Meta

Journal des traducteurs

Translators' Journal

\title{
La traduction et la constitution des langues : le cas de l'arabe
}

\section{André Roman}

Volume 35, numéro 1, mars 1990

Actes du colloque international " La traduction proligère "

URI : https://id.erudit.org/iderudit/003973ar

DOI : https://doi.org/10.7202/003973ar

Aller au sommaire du numéro

Éditeur(s)

Les Presses de l'Université de Montréal

ISSN

0026-0452 (imprimé)

1492-1421 (numérique)

Découvrir la revue

Citer cet article

Roman, A. (1990). La traduction et la constitution des langues : le cas de l'arabe.

Meta, 35(1), 195-206. https://doi.org/10.7202/003973ar d'utilisation que vous pouvez consulter en ligne.

https://apropos.erudit.org/fr/usagers/politique-dutilisation/ 


\title{
LA TRADUCTION ET \\ LA CONSTITUTION DES \\ LANGUES : LE CAS DE L'ARABE
}

\author{
ANDRÉ ROMAN \\ Université Lumière-Lyon II, Lyon, France
}

\section{LA TRADUCTION DU MONDE}

Lorsque l'Homme a su inventer dans son environnement et pour sa relation à son environnement les entités du monde auxquelles il pouvait dès lors prêter une identité, il s'est donné leur mémoire en les nommant.

Cette nomination par lui des entités qu'il inventait est authentiquement une traduction.

L'Homme a su inventer des entités lorsqu'il a été capable d'inventer une combinatoire. De fait, aucune invention n'est possible que par la mise en œuvre d'une combinatoire de composantes.

L'Homme, donc, inventeur de combinatoires, a imaginé dans le monde des res et des modus: des res, c'est-à-dire des entités imaginées par lui hors du temps, comme étrangères au temps, dont le temps n'est pas une composante; exemple: homme; des modus, c'est-à-dire des entités imaginées par lui dans le temps, comme s'inscrivant dans un déroulement apparent du temps, dont le temps est l'une des composantes; exemple: vivre.

\section{LA CONSTITUTION DES LANGUES}

Les noms que l'Homme devait inventer parallèlement sont les images linguistiques des res et des modus du monde ${ }^{1}$.

La combinatoire linguistique à laquelle l'Homme a eu recours pour nommer res et modus a été, naturellement, une combinatoire vocale.

\section{LA CONSTITUTION DE LA LANGUE ARABE}

La combinatoire fondamentale de la langue arabe, des langues sémitiques, est une combinatoire de consonnes. Cette combinatoire semble être née d'un ancien stéréotype laryngal, présémitique, un stéréotype tendu, qui aurait réalisé chaque voyelle $/ \mathrm{V} / \mathrm{comme}$ une voyelle préglottalisée: $\left[{ }^{?} \mathrm{~V}\right]$.

Et c'est l'instabilité des voyelles préglottalisées après consonnes sonores qui aurait produit le système syllabique ainsi conditionné phonétiquement:

$$
\mathrm{S}=\{\mathrm{CV}, \mathrm{CVC}\}
$$

qui est le système syllabique typique des langues sémitiques et que la langue arabe classique a conservé.

\section{A. LE SYSTĖME DE NOMINATION DE LA LANGUE ARABE}

Un tel système syllabique détermine une disjonction du sous-ensemble des consonnes, $\{\mathrm{C}\}$, et du sous-ensemble des voyelles, $\{\mathrm{V}\}$ :

$$
\mathrm{S}=\{\mathrm{CV}, \mathrm{CVC}\} \Rightarrow\{\mathrm{C}\} \cap\{\mathrm{V}\}=\varnothing
$$


Cette disjonction, dès lors que les consonnes et les voyelles peuvent être utilisées indépendamment les unes des autres, permet l'attribution systématique de tâches différentes aux consonnes et aux voyelles. C'est ainsi que s'est trouvée constituée la structure fondamentale des langues sémitiques.

En effet les langues sémitiques ont construit leur système de nomination sur des racines de consonnes.

Et c'est par un recours au même modus operandi qu'elles ont, pour développer encore leurs systèmes de nomination, employé une combinatoire partielle de leurs racines de consonnes.

Quant aux éléments du sous-ensemble complémentaire du sous-ensemble des consonnes, c'est-à-dire les voyelles, celles d'entre elles qui sont brèves étaient et, en arabe classique, sont encore utilisées, à l'exclusion des consonnes, comme des fonctionnels désinences.

L'organisation générale de l'arabe, des langues sémitiques, peut donc être représentée ainsi :
$\mathrm{S}=\{\mathrm{CV}, \mathrm{CVC}\}=>$

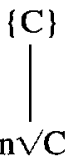
Système de nomination
ก

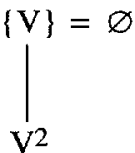
Système de communication

Ainsi chaque unité de nomination apparaît accompagnée d'une unité de communication qui l'établit dans la phrase.

En conséquence, la langue n'aura guère eu à faire recours à l'ordre et non plus à l'intonation qui sont les autres ressorts de la syntaxe.

Précisément les unités de nomination particulière ont été construites, systématiquement, sur des racines de trois consonnes parce que seule la combinatoire de trois consonnes pouvait produire en nombre suffisant les arrangements constituant les racines, c'est-à-dire les premiers signifiants de leurs signifiés particuliers.

Cependant si les unités de nomination particulière, spécifiées sémantiquement, comme celles, par exemple, dénotant la res $/ \mathrm{kalb} /,=$ «chien», de racine $\vee \mathrm{k}-\mathrm{l}-\mathrm{b}$, ou le modus /dari:b/, = «frappeur», de racine $\vee \mathbf{d}-\mathrm{r}-\mathrm{b}$, devaient, en raison d'une nécessité sémantique évidente qui imposait qu'elles fussent nombreuses et différentes, être construites sur des racines de trois consonnes, les unités de nomination non spécifiées sémantiquement ou unités de nomination générale, elles, peu nombreuses, pouvaient être construites sur des racines d'une seule consonne. Ces unités de nomination générale ne désignent jamais qu'une entité qui est soit unique - exemple: je —, soit montrée comme unique - exemple : ceci -, soit encore non différenciée - exemple latin : quid.

Celles des unités de la langue arabe qui sont construites sur des racines sont ses unités fléchies. Celles de ses unités qui ne sont pas construites sur des racines sont ses unités amorphes: leurs signifiants ne varient pas.

\section{LES UNITÉS FLÉCHIES}

La combinatoire des racines entre elles est visualisée par le tableau ci-après dans lequel $\alpha=1$ ou, exceptionnellement, $2 ; \beta=1$ ou 2 ou 3 : 
TABLEAU GÉNÉRAL DES UNITÉS FLÉCHIES

\begin{tabular}{|c|c|c|}
\hline \multicolumn{3}{|c|}{$+\vee C$} \\
\hline $\begin{array}{l}\text { UNITÉS DE NOMINATION } \\
\text { GÉNÉRALE }\end{array}$ & \multicolumn{2}{|c|}{$\begin{array}{l}\text { UNITÉS DE NOMINATION } \\
\text { PARTICULIÈRE }\end{array}$} \\
\hline$\vee C$ & \multicolumn{2}{|c|}{ VCCC } \\
\hline VC & $\begin{array}{l}1 \\
\text { VCCC } \\
{[\text { res }]}\end{array}$ & $\begin{array}{c}\text { vCCC } \\
{[\text { modus }]}\end{array}$ \\
\hline $\begin{array}{l}1 \\
\alpha \vee C\end{array}$ & & $\beta \vee C$ \\
\hline
\end{tabular}

Les unités de nomination construites sur une ou plus d'une racine monoconsonantique, qui sont des unités de nomination générale, sont les pro-formes de la langue.

Les unités construites au moins sur une racine triconsonantique, qui sont des unités de nomination particulière, sont ses formes.

\section{a) LES UNITÉS DÉNOTANT DES RES}

(1) Les unités dénotant des res et construites sur une seule racine, $\vee C$

Exemples:

- La pro-forme, de racine $\sqrt{ } \mathrm{n}$, du lieu général ou pro-lieu, $/ \mathrm{n} /$, signifiant d'un lieu, donc, réel ou figuré, qui n'est pas spécifié.

- La pro-forme $/ \mathrm{ma}: /,=$ «que ?» «quoi ?», de racine $\vee \mathrm{m}$ dénotant la res générale.

Les personnes et les non-personnes $/$ ?ana $: /=\langle\mathrm{je} » /, /$ ?anta $/=\langle\mathrm{tu} », / \mathrm{ka} /=\langle\mathrm{toi} », \ll \mathrm{de}$ toi $», /$ hu $/=\ll$ le», «de lui», de racines, $\vee$ $?, \sqrt{ }$ t, $\vee$ k et ${ }^{*} \vee \mathrm{c}$.

(2) Les unités dénotant des res et construites sur deux racines monoconsonantiques Exemple:

- La pro-forme de lieu / ?anna:/, = «où ?», construite sur $\vee \mathrm{n}$ et $\vee \mathrm{m}$.

(3) Les formes dénotant des res construites sur une racine triconsonantique

Dans la protolangue arabe, les res de racine triconsonantique étaient construites sur le paradigme $R_{1} V_{1} R_{2} \varnothing R_{3}$ caractérisé comme un paradigme de res par l'absence de voyelle, ou voyelle $\varnothing$, entre $R_{2}$ et $R_{3}$. Dans ce paradigme, $V_{1}$, entre $R_{1}$ et $R_{2}$, était le signifiant de la modalité d'animéité ${ }^{3}$.

\section{b) LES UNITÉS DÉNOTANT DES MODUS}

La modalité essentielle des modus est l'aspect, qui est une certaine traduction du temps, qui définit le déroulement intrinsèque de chaque modus ainsi considéré en lui-même. 
Trois autres modalités étaient portées par la forme même du modus sans être des modalités du modus même: la diathèse, le mode et l'agentivité.

Dans la protolangue arabe les unités dénotant des modus de racine triconsonantique étaient fondées sur le paradigme $R_{1} V_{1} R_{2} V_{2} R_{3}$ caractérisé comme un paradigme de modus par la présence de la voyelle $V_{2}$, entre $R_{2}$ et $R_{3}$. Dans ce paradigme, $V_{1}$, entre $R_{1}$ et $\mathbf{R}_{2}$, était le signifiant de la modalité de diathèse.

La voyelle $\mathrm{V}_{2}$, elle, était le signifiant de la modalité d'agentivité qui surdéterminait la diathèse subjective. Ou bien le sujet du modus était sans pouvoir sur lui, ou bien il participait à la production du modus, soit par son action spontanée, volontaire, soit par son action provoquée, par réaction. Ainsi, dans la diathèse subjective, $V_{2}$, entre $R_{2}$ et $R_{3}$, était le signe de l'appartenance de la forme à l'ensemble des modus et le signifiant de l'agentivité. Dans la diathèse objective, comme celle-ci implique une agentivité nulle, $V_{2}$ ne servait plus qu'à dénoter l'appartenance de la forme à l'ensemble des modus.

Les unités dénotant des modus pouvaient être construites sur une ou deux ou trois ou quatre racines.

Quatre modus seront cités :

Le modus de racine * $\vee c$, qui est le seul modus de racine monoconsonantique, de sens être ou faire.

- Le modus determinans, originellement de paradigme $\vee R_{1} a R_{2} v: R_{3}$. Ce modus impersonnel était caractérisé par la modalité aspectuelle de non-achèvement.

Exemples:

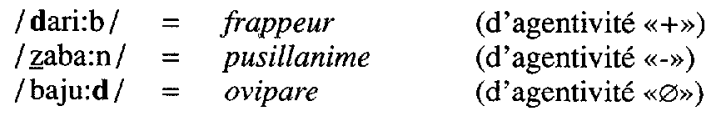

le modus personnel de paradigme $\mathrm{R}_{1} \mathrm{vR}_{2} \mathrm{R}_{3}+\mathrm{Rv}$ du verbe de modalité aspectuelle achevé.

Exemple:

$$
\mid \text { darab }- \text { ta } /=\text { Tu as frappé, battu. }
$$

- le modus personnel, ou verbe, de paradigme $\mathrm{R}_{1} \mathrm{vR}_{2}: \mathrm{R}_{3}+\mathrm{Rv}$. Exemple:

/ dar:ab- ta $/=\quad$ Tu as frappé à plusieurs reprises, $t u$ as battu (les cartes)

où la longueur de $\mathrm{R}_{2}$ était le signifiant de l'itération.

\section{LES UNITÉS AMORPHES DU SYSTĖME DE NOMINATION}

Les voyelles dont le système syllabique impose l'emploi ont été utilisées par le système de nomination comme les premiers signifiants de ses modalités. Ces voyelles, qui sont des éléments constitutifs des formes auxquelles elles appartiennent, sont de ce fait des unités liées. Les modalités libres, elles, comportent au moins une syllabe, /CV/ ou $/ \mathrm{CVC} /$, qui est le seuil de leur liberté syntagmatique. Certaines de ces modalités sont des phrases, les interjections de la Tradition. 
TABLEAU DES UNITÉS AMORPHES

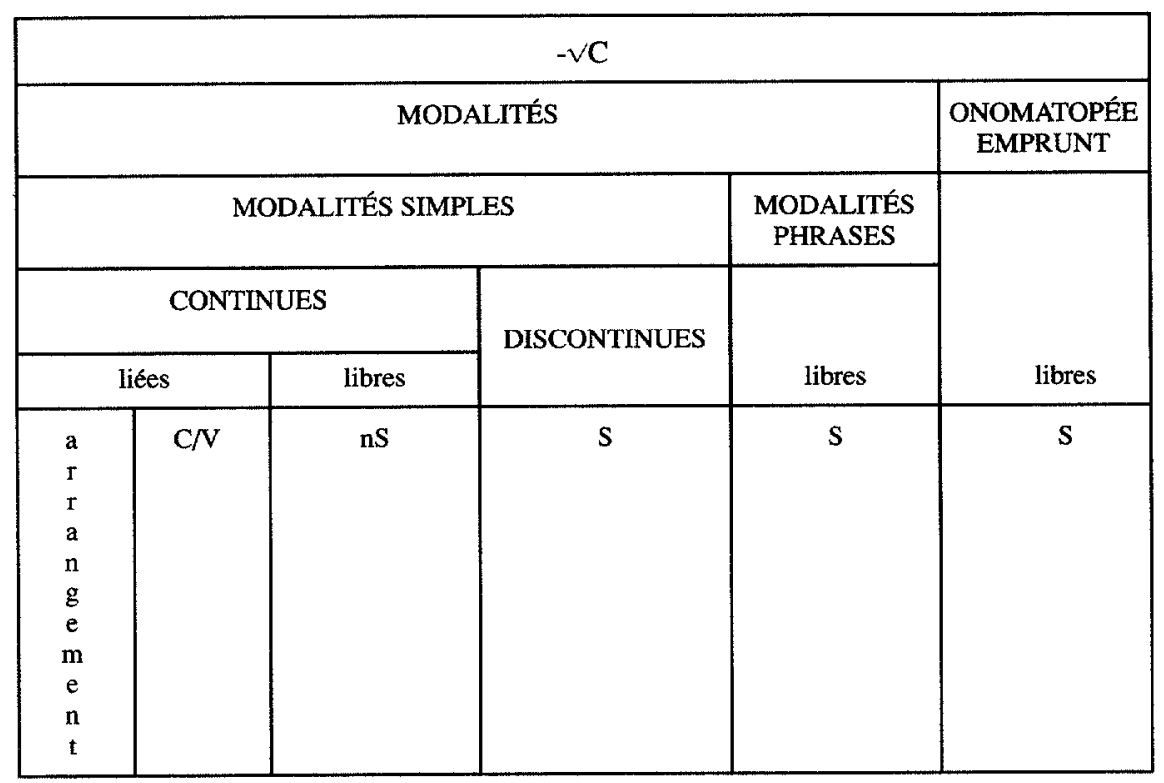

\section{B. LE SYSTĖME DE COMMUNICATION DE LA LANGUE ARABE}

La phrase est l'unité maximale du sỳstème de communication. Deux éléments étant nécessaires et suffisants pour constituer un système, le noyau de la phrase est constitué comme un système simple par deux seuls éléments. La relation entre ces deux constituants élémentaires est une relation structurelle fondamentale, immanquablement présente, la traduction de leur contrainte réciproque d'existence. Et la phrase se sera développée, toujours, par des extensions des constituants de son noyau et des extensions de ces extensions mêmes ${ }^{4}$.

\section{Le plan de la phrase}

Le système de communication, c'est-à-dire le système constituant les unités de nomination en phrases, s'est établi sur le plan suivant de la phrase:

\section{PLAN DE LA PHRASE}

\begin{tabular}{|cc|}
\hline $\mathrm{x}<$ & $\mathrm{y}$ \\
\hline$+/ \mid$ & $+/ \mid$ \\
$\mathrm{x}^{\prime}$ & $\mathrm{y}^{\prime}$ \\
$+/ 1$ & $+/ \mid$ \\
$\mathrm{x}^{\prime \prime}$ & $\mathrm{y}^{\prime \prime}$ \\
$\cdot$ & $\cdot$ \\
$\cdot$ & $\cdot$ \\
\hline
\end{tabular}

\{ NOYAU

[ EXTENSIONS 
Dans ce plan, les unités de nomination $x$ et $y$ sont les deux éléments fondamentaux, structurellement nécessaires et inomissibles, du noyau de la phrase, ses éléments nucléaires, reliés par une relation biunivoque de cooccurrence comme dans un duo, la première voix et la seconde voix. Et $x^{\prime}, y^{\prime} .$. sont les unités de nomination éventuellement appelées par le locuteur, selon ses besoins ; ce sont des unités sans aucune nécessité structurelle; $x^{\prime}, y^{\prime}$ sont des extensions reliées à leurs bases soit par la relation binaire de coordination, symbolisée par + , soit par la relation, également binaire, de subordination, symbolisée par |.

\section{Le duo des deux voix}

Le duo idéal des deux voix est le duo res - modus, cela parce qu'il établit par luimême la relation au temps qui est inhérente aux expériences du monde que les phrases verbalisent.

Exemples:

/darab-ta $/, \quad=$ Tu as frappé.

/ ?anta dari:b-u-n/ = Tu es frappeur.

\section{L'ÉQUILIBRATION DU SYSTÈME DE SYSTÈMES}

L'organisation générale, telle qu'elle a été dépeinte, en raison de sa structuration même, ne pouvait être parfaite. Aussi son équilibre ne pouvait-il être stable.

\section{A. L'ÉQUILIBRATION DU SYSTĖME PHONOLOGIQUE}

L'on a vu que le système syllabique qui a occasionné l'organisation générale des langues sémitiques serait né d'un conditionnement interne.

Ce conditionnement interne a détruit leurs voyelles préglottalisées, $\left[?{ }^{?} \mathrm{~V}\right]$, et produit leurs consonnes postglottalisées, [C?].

Dans le système phonologique ainsi constitué, d'autres transformations se sont produites, en raison de son déséquilibre ; mais elles n'ont pas affecté la structuration générale des langues sémitiques naissantes. Particulièrement, les consonnes postglottalisées, /C? / sont devenues des consonnes emphatiques.

\section{B. L'ÉQUILIBRATION DU SYTĖME DE NOMINATION}

\section{Les faits physiologiques}

L'intonation, qui est également un phénomène physiologique, comme elle est omniprésente dans l'acte de parole, s'est posée en concurrente de certaines modalités.

Exemple:

$$
\text { O Zayd! = /ja: zajd } />/ \text { zajd } /=\text { Zayd! }
$$

où la modalité d'appel, /ja:/, faite de phonèmes, cède la place à une modalité faite d'intonèmes.

\section{Les faits d'analogie}

L'analogie peut produire une nouvelle régularité, partielle et non plus générale, qui est destructrice du système.

Exemples :

- Dans le protoparadigme de l'achevé, la première personne du singulier, /darab - tu/ = $J$ 'ai frappé, était réalisée ${ }^{*} / \mathrm{darab}-\mathrm{ku} /$ avec un morphème de personne de racine ${ }^{*} \mathrm{v}$ et la voyelle syntagmatique $/ \mathrm{u} /$, homophone de $/ \mathrm{k} / ;$ l'unification du paradigme sur les deuxièmes personnes, /darab - t.../, a fait de la voyelle /u/le signifiant de la première personne: une voyelle est ainsi devenue radicale! 
Dans la conjugaison, un certain nombre de verbes ont été construits secondairement sur des racines, hors système, de quatre consonnes.

Ces verbes, toujours plus nombreux, ont été inventés dans les paradigmes à modalité d'itération qui a pour signifiant l'allongement de $\mathrm{R}_{2}$ : le segment constitué par cette consonne allongée a été recomposé en un segment de même quantité occupé par deux consonnes différentes; d'où $\sqrt{ } R_{1} R_{2}: R_{3}>\sqrt{ } R_{1} R_{2} R_{3} R_{4}$. La conjugaison de ces verbes quadriconsonantiques est celle même des verbes triconsonantiques sur lesquels ils sont calqués.

Exemples:

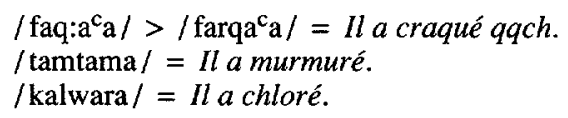

Plus généralement, si certains paradigmes ont pu être construits idéalement, leurs phonèmes participant, tous, d'un signifié, tels les schèmes de l'achevé et de l'inachevé du verbe simple ou du nom masculin, d'autres ne pouvaient l'être. Ainsi, par exemple, les verbes quadriconsonantiques qui viennent d'être cités. Ainsi le nom féminin qui ne pouvait être construit qu'avec une voyelle syntagmatique, c'est-à-dire avec un phonème sans relation aucune avec un signifié. D'où

$$
/ \text { kalb-a-t } /=\text { chienne }
$$

avec, entre $/ b /,=R_{3}$, et $/ t /$, signifiant du féminin, une voyelle, /a/, syntagmatique.

En effet, le système syllabique

$$
\mathrm{S}=\{\mathrm{CV}, \mathrm{CVC}\}
$$

comme il produisait le système de systèmes qui a été reconnu, imposait le recours à des voyelles syntagmatiques.

Or les voyelles syntagmatiques, en altérant les schèmes dans lesquels elles entraient nécessairement, préparaient la transformation des racines consonantiques, $\vee C$, en racines syllabiques ou «radicaux», $\vee S$.

Une extension analogique du modus operandi de la langue, par combinatoire de racines, allait produire, secondairement, des unités de nomination composées d'une forme et d'une racine. Deux combinaisons symétriques allaient ainsi être produites :

- la combinaison d'une forme dénotant un modus et de la racine de la res générale réalisée $/ \mathrm{t} / /^{5}$.

Exemple :

$\{$ dari: $b+\mathfrak{t}\}>/$ dari:bat $/=$ redevance, impôt $(<\mathrm{la}$ res frappeuse $)$

la combinaison d'une forme dénotant une res et de la racine du modus général, * $\vee c$, réalisée $/ \mathrm{jj} /$.

Exemples:

$\{\mathrm{kal} \varnothing \mathrm{b}+* \sqrt{ } \mathrm{c}\}>/ \mathrm{kalb}-\mathrm{i}-\mathrm{jj} /=$ canin, cynique $(<$ être chien $)$
$\{\mathrm{bah} \varnothing \mathrm{r}+* \sqrt{ } \mathrm{c}\}>/ \mathrm{bahr}-\mathrm{i}-\mathrm{jj} /=\operatorname{marin} \quad(<$ être mer $)$

C'est là une autre rupture du système. En effet ces unités de nomination ont été créées par transformation d'une forme en un radical, c'est-à-dire en une séquence opacifiée de phonèmes où les consonnes radicales elles-mêmes n'ont d'identité qu'empruntée à l'histoire de la langue ${ }^{6}$. 


\section{Les faits de concurrence}

Les modalités qui sont mortes, particulièrement l'itération, l'agentivité, l'animéité, sont mortes de la concurrence des compléments. En effet ceux-ci constituent des moyens d'un emploi de beaucoup plus précis.

La concurrence du système de communication a donc produit dans le système de nomination, son «jumeau», avec lequel il est organiquement lié, des altérations profondes.

\section{L'ÉQUILIBRATION DU SYSTÈME DE COMMUNICATION}

\section{Les faits d'analogie}

C'est une analogie sémantique qui a produit la phrase nominale.

En raison de sa persistance même, le modus determinans était susceptible d'apparaître comme exprimé hors du temps. Cette fausse apparence a fait qu'à une certaine étape de l'évolution de la langue, l'aspect qu'il dénote n'a plus été reconnu: le modus determinans a été alors perçu comme une autre figure du nom, comme une res. Lorsque sa seconde voix, le modus determinans /dari:b/, a été identifiée comme étant une res, la phrase

$$
\text { / ?anta dari:b-u-n/ = Tu es frappeur }
$$

ou toute autre phrase pareille, est apparue comme inaugurant un paradigme de phrases dans lequel la relation nucléaire reliait non pas une res et un modus mais deux res.

Exemple :

/ ?anta marø?-u-n/ = Tu es un homme.

\section{Les faits de concurrence}

L'intonation a pris le pas, dans la langue arabe moderne, sur les fonctionnels non spécifiés sémantiquement qui sont pourtant les fonctionnels fondamentaux de la langue, ses fonctionnels désinences $\mathrm{V}$.

Exemple :

D'où la chute, fréquente, de ces voyelles désinences.

/darab-ta zajd-a-n $/>>$ darab-ta zajd $/=T$ u as frappé Zayd.

\section{L'ÉQUILIBRATION DES SYSTĖMES DE NOMINATION ET DE COMMUNICATION}

La chute des voyelles désinences a souvent produit des syllabes anomales. Exemple repris :

/ darab-ta zajd/ = Tu as frappé Zayd.

où / zajd/ est réalisé CVCC.

Or, on l'a vu, le système syllabique d'où est née la langue arabe ne comptait que les deux syllabes CV et CVC. Toute modification de ce système, comme elle abolit la disjonction des deux sous-ensembles des consonnes, $\{\mathrm{C}\}$, et des voyelles, $\{\mathrm{V}\}$, détruit la base même de l'organisation de la langue arabe.

\section{PROBLÉMATIQUE DE LA CRÉATION LEXICALE}

L'on a vu que la langue arabe, dans sa nomination des entités du monde, signifiait, globalement, par la racine, $\vee \mathrm{CCC}$, les traits particuliers à la morphologie de chacune d'elles et qu'elle signifiait, jadis, au moyen de modalités créées expressément, les seuls traits génériques: l'«animéité» et le «sexe» pour les res, l'aspect pour les modus. 
Dans $/ \mathrm{kal} \varnothing \mathrm{b} /,=$ chien, par exemple, la langue arabe signifiait, par la réalisation $\varnothing$ de $\mathrm{V}_{2}$, que cette unité de nomination dénotait une res; par la réalisation /a/de $\mathrm{V}_{1}$, que cette res était une res animée; par le silence même de la forme, que cette res était du sexe masculin; enfin, globalement, elle signifiait par la racine $\sqrt{ } \mathrm{k}-\mathrm{l}-\mathrm{b}$, les traits particuliers à la morphologie de l'animal : mammifère, digitigrade...

Très remarquable est la disproportion entre ce que le système de nomination peut dire détaillément et ce qu'il dit globalement.

Autrement dit, très remarquable est l'extension extrême de l'opération de réduction des res et des modus du monde que réalise la langue dans son opération de nomination.

L'évolution des langues suit la pente de cette réduction sémantique.

\section{Le renouvellement formel}

Aussi, par la force des choses, l'opération de nomination n'est-elle pas restée confinée dans les seules formes du système de nomination: elle a combiné, linéairement, des formes différentes dans une même unité terminologique qu'elle a ainsi constituée en un syntagme, c'est-à-dire en un constituant de phrase fait de $n$ unités de nomination.

\section{Le renouvellement sémantique}

Quant au renouvellement sémantique, là où il serait nécessaire, ce sont la métaphore, la métonymie et l'hypallage qui peuvent le produire.

Exemples:

$$
\begin{aligned}
& \text { Souris (d'ordinateur) } \\
& \text { nomen = créance }
\end{aligned}
$$

où c'est par métonymie du nom, nomen, inscrit sur les livres de compte au regard de la somme prêtée ou empruntée, que le mot est devenu, en latin, un terme et a pris le sens de créance.

Réseau despotique qui nomme un réseau synchronisé dans lequel une horloge maîtresse détient le pouvoir sur toutes les autres horloges.

\section{LA TRADUCTION DES EMPRUNTS, DES TERMES ET DES LANGUES}

\section{A. LA TRADUCTION DES EMPRUNTS}

La langue arabe inventant une racine ou empruntant à une autre langue une racine ne rompt pas avec son ancien système de nomination. La traduction n'aura eu, dans ce cas, d'autre effet que d'augmenter sa capacité lexicale.

C'est dans le seul cas d'un emprunt d'une unité de nomination réfractaire à l'arabisation que l'effet de l'emprunt pourra porter sur la constitution même de la langue.

Si l'unité de nomination empruntée ne peut être reconstruite sur une racine, elle prendra place parmi les unités amorphes, dans une nouvelle catégorie ad hoc où elle retrouvera les onomatopées qui sont la traduction phonologique et syllabique de bruits du monde restés dans la langue des éléments intrus.

Immanquablement cette nouvelle catégorie d'unités amorphes concurrencera la catégorie des unités de nomination de la langue constituées, conformément à son système, sur des racines de consonnes.

Exemple :

/ kat.ku:t/ = volaille caquetante, poulet

L'on voit bien qu'il y a là l'émergence d'un autre système de nomination construit, lui, sur des racines de syllabes.

Cependant le recours, par une langue, à deux systèmes de nomination, ne peut être que transitoire. 
Ou bien la langue reviendra à son système ancien, au demeurant non pas d'ellemême, ou bien, plus probablement, elle basculera, progressivement, dans son nouvel état.

$\mathrm{Si}$, en outre, l'unité étrangère est empruntée avec une forme syllabique étrangère, c'est alors le système syllabique propre à la langue arabe qui sera lui-même atteint. Exemple:

/ ful.klu:r/ $=$ folklore $^{8}$

Or plusieurs des syllabes étrangères à l'arabe n'apparaissent pas étrangères aux Arabes. En effet, leurs langues maternelles, qui sont les langues régionales du monde arabe, ses dialectes, ont des systèmes syllabiques fort proches du système syllabique des langues occidentales. Ainsi le hassânîya a le système syllabique suivant:

$\mathrm{S}=\{\mathrm{CV}, \mathrm{CCV}, \mathrm{CVC}, \mathrm{CVCC}, \mathrm{CCVC}, \mathrm{CCVCC}, \mathrm{V}, \mathrm{VC}, \mathrm{VCC}\}$

Or le système syllabique propre à l'arabe :

$\mathrm{S}=\{\mathrm{CV}, \mathrm{CVC}\}$

est le fondement même de l'organisation générale de la langue arabe.

Les perturbations profondes entraînées dans la langue par l'accroissement de son système syllabique sont manifestes dans les langues arabes régionales. Elles n'ont pas le système syllabique de l'arabe et elles n'ont pas son système de communication: les voyelles désinentielles ont dans ces langues totalement disparu et, en conséquence, l'ordre et l'intonation y jouent un rôle important.

\section{B. LA TRADUCTION DES TERMES}

Les termes techniques complexes sont réalisés ou bien par des syntagmes, ou bien par des affixes.

Les termes techniques modernes sont souvent complexes et différents des termes qui les ont précédés par le nombre de leurs affixes et encore par le fait que ceux-ci peuvent être soumis à un ordre lui-même contraint, significatif.

Les termes réalisés par des syntagmes, l'arabe peut les produire, d'emblée, conformément à ses systèmes de nomination et de communication.

Exemple:

/sabakat istibda:dijjat $/=$ réseau despotique ${ }^{9}$

Les termes qui comprennent de nombreux affixes, la langue arabe doit les traduire tels quels avec leurs affixes car ceux-ci leur'sont indispensables. Mais la langue arabe $s^{\prime}$ 'est constituée avec un nombre réduit de préfixes et de suffixes ${ }^{10}$. Et les nouveaux affixes arabes, transformés d'unités libres de la langue ou empruntés, ont changé, changent en unités opaques et, par là, en radicaux, les unités de nomination auxquelles ils sont raboutés.

Assurément ces termes peuvent être traduits en arabe. Ils ne peuvent être arabisés.

La langue arabe s'est donné, aujourd'hui, pour traduire ces termes, la majorité des affixes nécessaires.

Exemples :

$/$ tah - bahr $-\mathrm{ijj} /=$ sous-marin

où / tah / est l'abréviation de / tahta/ = sous; où /bahr-ijj/ est fait de $/ \mathrm{bahr} /=m e r$, et de /ijj/ où / i / est une voyelle syntagmatique et / jj/ l'avatar du modus général * $/ \mathrm{c}$.

$$
\text { /sukkar }-\mathrm{i}: \mathrm{d} /=\text { glucoside }
$$

où / sukkar/ est le nom arabe, très ancien, du sucre, et /i:d/ est le calque de ide. 
Ces affixes nouveaux, à l'instar des affixes secondaires anciens de la langue, métamorphosent son système de nomination.

\section{LA TRADUCTION DES LANGUES}

Que le terme technique ainsi constitué soit traduit d'une langue ou soit inventé, immédiatement, à partir du référent même est indifférent.

La traduction en arabe d'un texte étranger qui ne renferme point de tels termes n'a pas ces conséquences. Sans doute influencera-t-elle la phraséologie de l'arabe. Mais, semble-t-il, dans le sens de son évolution propre.

Trois exemples sont avancés, souvent, de l'influence de la traduction en arabe de langues occidentales.

Le premier exemple est celui du "complément d'agent».

La base du complément d'agent est nécessairement un modus de diathèse objective. Ce modus était donc d'agentivité nulle. Or l'agent, d'évidence, veut une agentivité non nulle. L'agentivité non nulle est de la seule diathèse subjective. Le complément d'agent était donc impossible dans le système de nomination de la langue arabe. Aussi le système de communication n'avait-il, dans le paradigme de ses fonctionnels, aucun fonctionnel qui aurait eu pour rôle d'introduire ce complément. Puis le complément d'agent est devenu possible quand la modalité d'agentivité est morte. Son impossibilité ancienne, cependant, s'est maintenue comme une norme de l'usage. Ses premières attestations sont rhétoriques. La traduction des langues occidentales aura hâté sa banalisation. Mais banalisation n'est pas rupture. La nouveauté n'est que dans l'effacement de l'effet rhétorique : l'usage rhétorique ancien s'est banalisé.

Le deuxième exemple touche à l" «expansion annective», le «complément de nom». L'on doit dire en bonne langue:

/bajøt-u 1 marø?-i wa kaløb-u hu /

La maison de l'homme et son chien

L'on peut dire désormais :

/ bajøt-u wa kaløb-u 1 mar $\varnothing ?-\mathbf{i} /$

La maison et le chien de l' homme

Là encore l'on trouve des attestations anciennes et rhétoriques de la seconde construction. Lorsque le tanwîn n'a plus été identifié comme la racine, $\vee \mathrm{n}$, du lieu général ${ }^{11}$, il est sorti du paradigme des compléments de nom. Le paradigme des compléments de nom s'est ainsi décroché de sa base qu'il n'était plus contraint de suivre immédiatement. Cependant, là encore, la contrainte ancienne du paradigme s'est maintenue comme une norme de l'usage.

Le troisième exemple concerne la traduction de «si» en phrase interrogative.

Si n'est plus signifié dans les phrases interrogatives par le morphème d'interrogation $/$ hal $/,=$ ?, mais par le morphème d'éventualité et d'hypothèse /?in/, l'équivalent même de si. Cet emploi récent de /?in/ ne constitue pas, non plus, une rupture : l'interrogation est affine à l'éventualité.

Exemple:

/natasa: ?alu ?in ka:nat ha:dihi t tari:qat/

Nous nous demandons si cette [méthode] était bien la méthode [à suivre].

\section{CONCLUSION}

Dans les conditions qui ont été examinées, le système de nomination ne pouvait plus créer de modalités nouvelles ni d'affixes nouveaux. 
Hors rupture, aucune création nouvelle du système de nomination n'était possible que de racines.

En revanche, les signifiés nouveaux pouvaient trouver leurs signifiants, toujours, dans des réalisations ad hoc du système de communication. Mais ces réalisations pouvaient être malcommodes ou refusées par l'usage international ${ }^{12}$.

Cependant l'intrusion dans les langues de termes qui doivent être des images plus précises qu'auparavant des référents qu'ils nomment accentue la pente de l'évolution des langues vers la réduction, symétrique, de leurs structures de nomination.

Dans le cas de la constitution de la langue arabe, ce phénomène aura été précédé par un autre phénomène : l'interférence avec les dialectes qui lui sont apparentés.

En effet l'exceptionnelle stabilité de la koinè arabe avait été assurée par plusieurs facteurs. Surtout il y a eu à partir du VIII ${ }^{\mathrm{e}}$ siècle, semble-t-il, la croyance des Arabes musulmans sunnites en l'institution divine de leur langue qu'ils ont dès lors considérée comme une langue dont l'Homme n'avait pas la propriété, sur laquelle il n'avait aucun pouvoir légitime, une langue intangible et donc arrêtée. Cependant les langues régionales, issues des langues des tribus arabes, elles, allaient évoluer librement, selon leurs pentes propres, en raison, d'abord, de leurs déséquilibres internes, pour devenir les langues vernaculaires des Arabes, les dialectes arabes.

Maintenant, les langues régionales concourent à la déstabilisation de la langue arabe en faisant apparaître comme arabes des structures syllabiques qui ne le sont aucunement.

Ce phénomène aura joué plus que la traduction d'autres langues.

\section{NOTES}

1. Le terme modus sera désormais utilisé pour modus linguistique; le terme res sera désormais utilisé pour res linguistique.

2. $n=1$ ou 2 ou 3 ou 4 .

3. Tout nom commun était, est à même de constituer avec la res $\vee \mathrm{n}$ de lieu un syntagme indéterminé, la res dénotée par le nom commun étant ainsi présentée comme non localisée. Le nom propre, qui est un nom généalogique, ne pouvait être apparié à vn car il nommait un membre d'une communauté qui, si elle n'était pas spécifiée, était impliquée par l'absence même de $\vee \mathrm{n}$. Ce sont les noms propres qui seraient à l'origine de la désinence à deux cas, $[/ \mathrm{u} /, / \mathrm{a} /]$, ou diptosie, qui affecte encore certains noms propres et, par analogie, certains noms communs.

4. En effet, le recours à des extensions du noyau est impossible; le noyau, comme il constitue une phrase ne pouvant en tant que phrase recevoir d'extension dès lors que la phrase est l'unité syntaxique maximale.

5. $/ \mathrm{t} / \mathrm{est}$ un allomorphe conditionné de $/ \mathrm{m} /$.

6. De fait, la voyelle $V_{2}$, entre $R_{2}$ et $R_{3}$, est soit /a/, soit / $i$ /, soit / $u$ /, dans les res dérivées d'une forme dénotant un modus, et elle est $\varnothing$ dans les modus determinans dérivés d'une forme dénotant une res.

7. Ou, avec la réalisation pausale classique : /darab-ta zajd-a : /.

8. Folklore avait d'abord été rendu par / ful.ku.lu:r/, - CVC.CV.CV., conformément au système syllabique.

9. Terme des télécommunications.

10. Les préfixes et les suffixes originels de la langue arabe sont les modalités qui, dans chaque unité fléchie, précèdent ou suivent les consonnes radicales.

11. Le tanwîn a été étudié dans la partie sur le système de nomination en 1. a) (1).

12. Il faut relever que la création de nouvelles figures des noms et les solutions de rupture font correspondre à un même champ référentiel des ensembles linguistiques différents. Autrement dit le lexique de ce champ n'est pas signifié par les membres d'une seule famille linguistique. La seule famille qui puisse en rester une est la famille référentielle. Une langue qui ne nommerait plus, régulièrement, les entités du monde avec les moyens reconnaissables d'un système de nomination, mais qui leur donnerait des noms opaques, qui apparaîtraient donc comme des signifiants globaux de leurs signifiés, cette langue laisserait face à face ses noms et leurs nommés. Dans une telle langue, le nom ne pourrait être défini linguistiquement, c'est-à-dire indépendamment de son référent. 